\title{
Endoscopic modified medial maxillectomy for odontogenic cysts and tumours*
}

\author{
Tsuguhisa Nakayama1,2, Nobuyoshi Otori², Daiya Asaka², Tetsushi Okushi², \\ Shin-ichi Haruna ${ }^{1}$
}

Rhinology 52: 376-380, 2014

DOI:10.4193/Rhino13.134

*Received for publication:

August 27, 2013

Accepted: March 17, 2014

\begin{abstract}
Background: Odontogenic maxillary cysts and tumours originate from the tooth root and have traditionally been treated through an intraoral approach. Here, we report the efficacy and utility of endoscopic modified medial maxillectomy (EMMM) for the treatment of odontogenic maxillary cysts and a tumour.
\end{abstract}

Methodology: We undertook EMMM under general anaesthesia in six patients: four had radicular cysts, one had a dentigerous cyst, and one had a keratocystic odontogenic tumour.

Results: The cysts and tumours were completely excised and the inferior turbinate and nasolacrimal duct were preserved in all patients. There were no peri- or postoperative complications, and no incidences of recurrence.

Conclusion: Endoscopic modified medial maxillectomy appears to be an effective and safe technique for treating odontogenic cysts and tumours.

Key words: endoscopic sinus surgery, keratocystic odontogenic tumour, radicular cyst, dentigerous cyst, maxillary sinus

\section{Introduction}

Odontogenic maxillary cysts and tumours arise in the maxilla and originate from the root of the tooth. In 2005, the World Health Organization classified keratocystic odontogenic tumour as a benign cystic neoplasm. Incomplete removal of the cystic lining may reflect technical difficulties encountered during excision, but risks recurrence ${ }^{(1)}$. Dentigerous cysts should also be completely resected ${ }^{(2)}$, while marsupialisation is considered to be acceptable for radicular cysts ${ }^{(3)}$. Nevertheless, it is sometimes difficult to discriminate between keratocystic odontogenic tumours and radicular cysts before surgery ${ }^{(4)}$; consequently our clinical practice is to completely excise the lesion in all such cases.

Traditionally, odontogenic maxillary cysts have been treated by marsupialisation, enucleation, or enucleation and bone grafting by means of an intraoral approach by dentists and oral and maxillofacial surgeons ${ }^{(3)}$. As the indications for endoscopic sinus surgery have expanded, there have now been several reports of odontogenic cysts and tumours being treated endoscopically $(2,5,6)$. However, it is not always possible to completely enucleate the lesion in every case using this technique alone.

Endoscopic medial maxillectomy (EMM) is a safe and effective procedure for the treatment of inverted papillomas originating from the anteromedial and anterior walls of the maxillary sinus $(7,8)$. Extensive turbinate resection, however, may result in abnormal nasal aerodynamics ${ }^{(9)}$. Some patients who have undergone EMM complain of empty nose syndrome ${ }^{(10)}$. Once the empty nose syndrome arises, it is very difficult to treat ${ }^{(11)}$.

We previously reported the use of endoscopic modified medial maxillectomy (EMMM) for the treatment of inverted papillomas and mucocoeles ${ }^{(12)}$. This technique completely preserves the inferior turbinate mucosa and nasolacrimal duct, and preserves as large a portion of the inferior turbinate as possible, while facilita- 


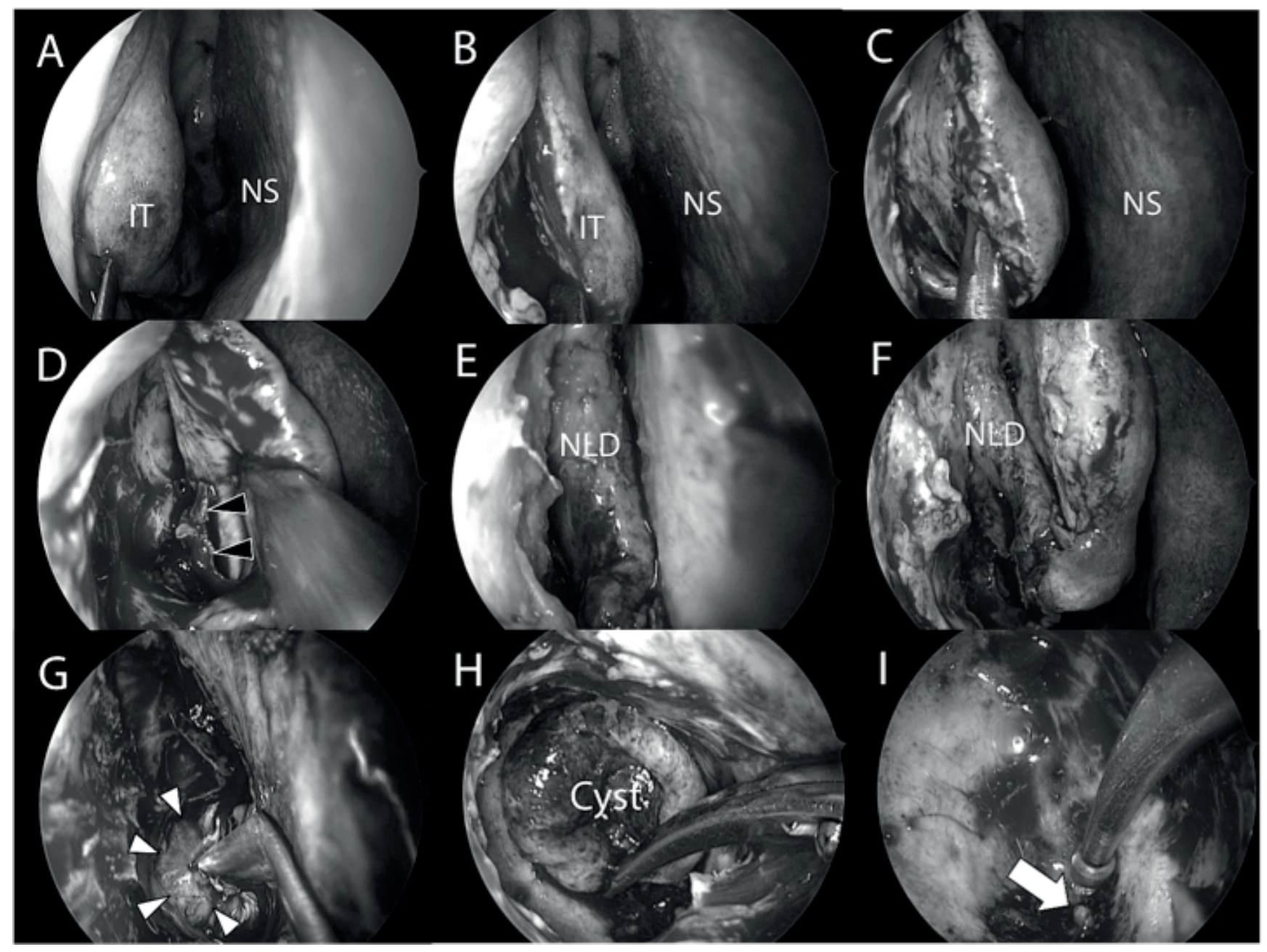

Figure 1. Intraoperative endoscopic photographs of endoscopic modified medial maxillectomy in a patient with a radicular cyst.

(A) Incision of the lateral wall parallel to the mucocutaneous junction directly behind the pyriform aperture. (B) Elevation of the nasal mucosa. (C) The bone of the inferior turbinate (IT) was adequately exposed. (D) A cut was made between the conchal crest and the inferior turbinate. The IT is visible (Black arrowheads). (E) After resection of the lateral nasal wall, the nasolacrimal duct (NLD) was widely exposed. (F) The nasal mucosa and NLD could be displaced medially. (G) Exposure of the radicular cyst (white arrowheads). (H) Endoscopic view $\left(70^{\circ}\right)$ inside the cyst. The cyst was clearly visible. (I) After cyst resection, the root of the affected tooth was visible (white arrow).

ting equally good access to the maxillary sinus as conventional EMM. We undertook a prospective study to establish the safety and efficacy of EMMM for the treatment of odontogenic cysts and tumours that occupy most of the maxillary sinus.

\section{Materials and methods}

Study design and patient population

We report a prospective case series of six patients who underwent EMMM between April 2011 and May 2012 at Jikei University Hospital, Tokyo, Japan, and Dokkyo Medical University Hospital, Tochigi, Japan. The ethics committees of both institutions approved the conduct of the study. Four patients had radicular cysts, one patient had a dentigerous cyst, and one patient had a keratocystic odontogenic tumour. One of the patients with radicular cysts had bilateral disease. In all patients, the lesion occupied most of the maxillary sinus.

\section{Surgery}

We performed EMMM under general anaesthesia as previously described ${ }^{(12)}$. First, we created a mucosal incision just behind the pyriform aperture (Figure $1 \mathrm{~A}$ ) and elevated the nasal mucosa from the lateral nasal wall (Figure 1B). Next, we adequately exteriorised the inferior turbinate (Figure $1 C$ ) and cut between the conchal crest and inferior turbinate with a chisel (Figure 1D). The lacrimal process of the inferior turbinate, frontal process of the maxilla, and inferior portion of the lacrimal bone were resected using a diamond burr. The nasolacrimal duct was then widely exposed (Figure 1E). We elevated the mucosa of the inferior meatus from the lateral nasal wall so that the nasal mucosa and nasolacrimal duct could be displaced medially (Figure 1F). The 
Table 1. Patient characteristics and outcomes of endoscopic modified medial maxillectomy.

\begin{tabular}{|c|c|c|c|c|c|c|}
\hline No. & Age (yrs) & Gender & Side & Disease & Follow-up (mo) & Current status \\
\hline 1 & 33 & $\mathrm{M}$ & Right & $\begin{array}{l}\text { Keratocystic odon- } \\
\text { togenic tumour }\end{array}$ & 32 & Disease free \\
\hline 2 & 66 & M & Right & Dentigerous cyst & 24 & Disease free \\
\hline 3 & 35 & M & Bilateral & Radicular cyst & 15 & Disease free \\
\hline 4 & 69 & $\mathrm{~F}$ & Left & Radicular cyst & 10 & Disease free \\
\hline 5 & 69 & M & Right & Radicular cyst & 17 & Disease free \\
\hline 6 & 40 & $M$ & Right & Radicular cyst & 6 & Disease free \\
\hline
\end{tabular}

mucosa of the inferior turbinate was sutured to the nasal septum if it obstructed the surgical field. Next, we drilled away the medial wall of the maxillary sinus with a diamond burr and identified the cyst (Figure 1G). We mostly used a $0^{\circ}$ endoscope, but a $70^{\circ}$ endoscope was used in the anterior wall of the cyst and root of the affected tooth (Figure $1 \mathrm{H}, \mathrm{I}$ ). Finally, the transposed nasal mucosa and nasolacrimal duct were replaced, and the anterior portion of the transposed nasal mucosa was sewn to the lateral nasal wall.

\section{Results}

Table 1 summarises the patients' characteristics and outcomes after EMMM. The cyst walls and keratocystic odontogenic tumour were completely excised, and the inferior turbinate and nasolacrimal duct preserved, in all patients. There were no intraoperative or postoperative complications. The affected teeth were preserved in all four patients with radicular cysts. A dentist extracted the affected tooth prior to endoscopic surgery in the patient with a keratocystic odontogenic tumour, and the affected tooth of the patient with the dentigerous cyst was removed during the EMMM procedure (Figure 2). The mean follow-up period was 17.4 months (range, 6-32 months), and there has been no evidence of recurrence in any of the patients. Postoperative endoscopic views and computed tomography scans confirmed that the inferior turbinate had been preserved in every case (Figure 3). All patients reported that nasal breathing was satisfactory and none reported dryness.

\section{Discussion}

The three most common cystic lesions in the jaw are radicular
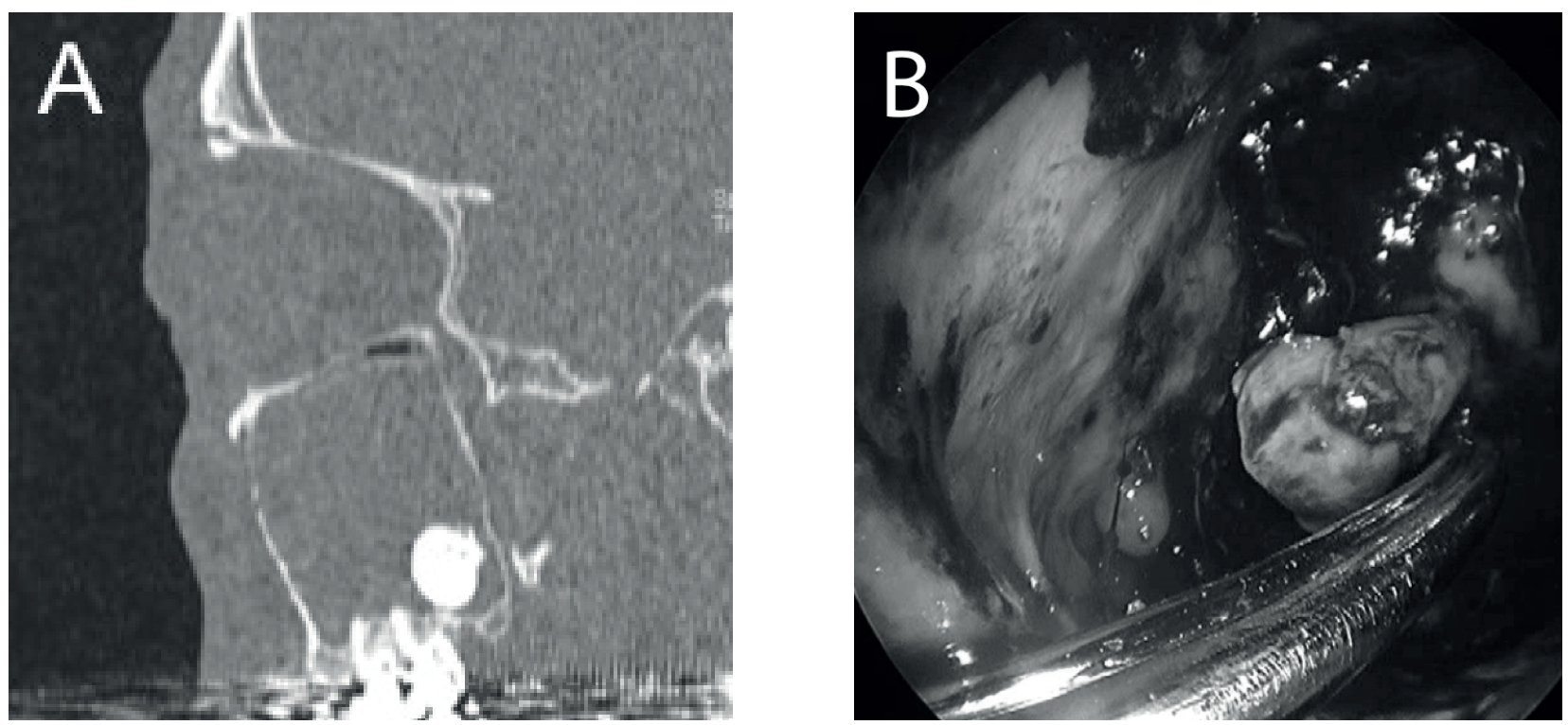

Figure 2. Images from Case 2. (A) Sagittal computed tomography scan showing cysts with the tooth occupying most of the maxillary sinus. (B) Endoscopic view $\left(70^{\circ}\right)$ inside the maxillary sinus showing the affected tooth. 

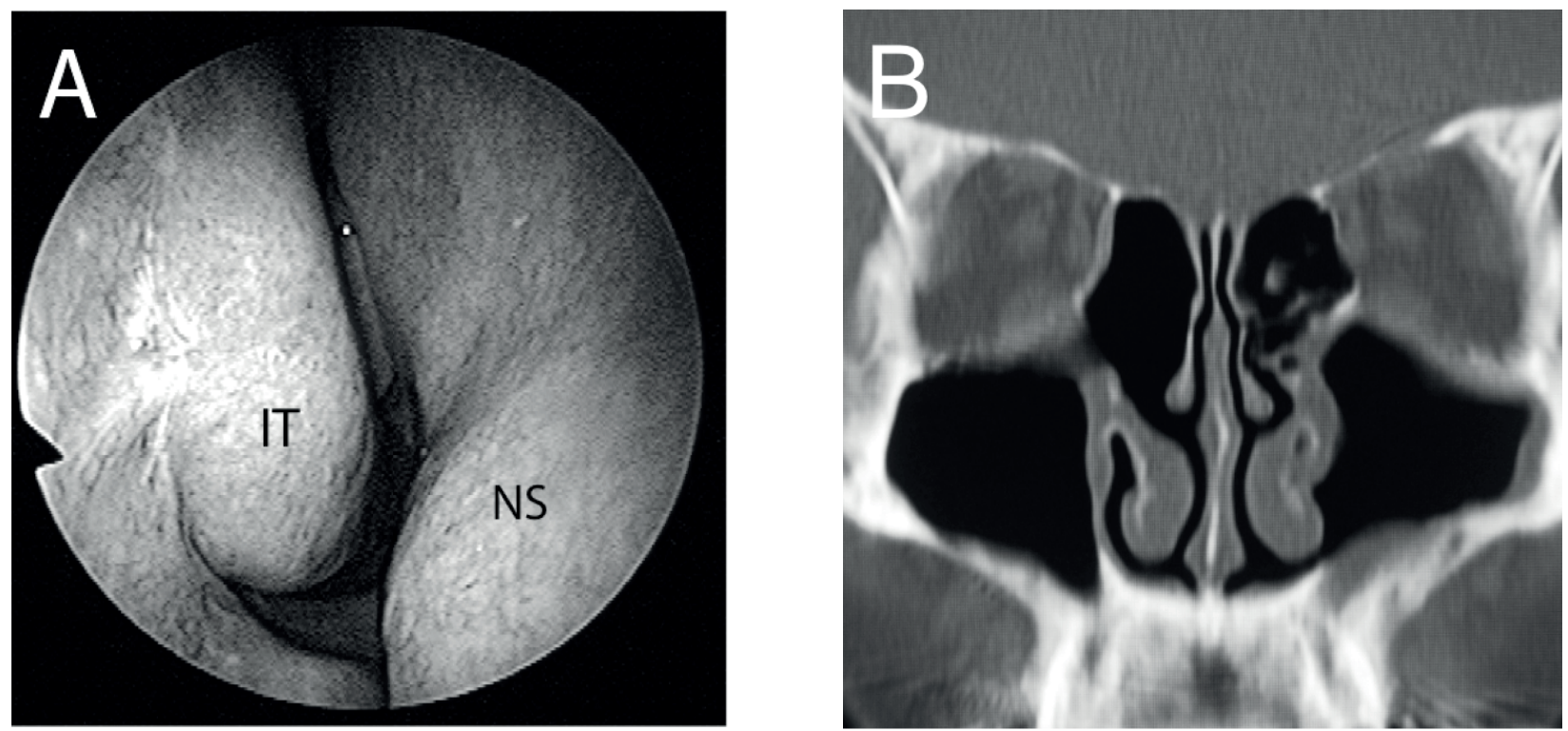

Figure 3. Images from Case 3. (A) Postoperative endoscopic view revealing preservation of the inferior turbinate 12 months after surgery. (B) Coronal computed tomography scan showing complete removal of bilateral radicular cysts and preservation of the inferior turbinate.

cysts, dentigerous cysts, and keratocystic odontogenic tumours (3). When these diseases become severe, they may extend into the maxillary sinus. Incomplete removal of the cystic lining of a keratocystic odontogenic tumour may be a consequence of technical difficulties during surgery, but is a risk factor for recurrence ${ }^{(1)}$. Furthermore, it may be difficult to differentiate between keratocystic odontogenic tumours and radicular cysts before surgery. Even if the lesion is diagnosed preoperatively as a radicular cyst, we recommend complete removal - particularly because malignant transformation has been reported ${ }^{(5,13-15)}$.

Odontogenic cysts and tumours have traditionally been treated via an intraoral approach by dentists and oral and maxillofacial surgeons. Otorhinolaryngologists have recently reported the successful endoscopic resection of odontogenic cysts and tumours when there is minimal extension into the maxillary sinus $(2,5,6)$. However, if the disease should occupy most of the maxillary sinus, enucleation is not possible. Staged operations have been reported for this type of extensive disease. First, a window in the cyst wall is created, and the cyst is decompressed. Complete resection is then carried out after the primary wound has closed ${ }^{(6,16)}$, but this staged approach is particularly onerous for patients.

The principal behind EMMM is to achieve access to the maxillary sinus comparable to that of conventional EMM while preserving the inferior turbinate and nasolacrimal duct. The difficulty with endoscopic resection of odontogenic cysts and tumours lies in accessing the anteromedial, anterior and inferior walls of the maxillary sinus, but these portions of the maxillary sinus can be reached using EMMM. In our experience, odontogenic tumours and cysts were relatively easy to resect using this technique, as it was possible to peel them away from the maxillary wall, which contrasts with our experience of inverted papillomas ${ }^{(12)}$. The advantage of using EMMM for odontogenic tumours and cysts is that it allows for complete resection in one operation. The use of EMM to treat these diseases has not been previously reported, but we believe that EMMM is superior as it allows the morphology of the nasal cavity to be preserved.

\section{Conclusion}

We used EMMM to excise several odontogenic maxillary cysts and one tumour in this case series of six patients. Endoscopic modified medial maxillectomy is a technique that allows the morphology of the nasal cavity and nasolacrimal duct to be preserved. Patient outcomes after surgery were excellent, confirming the safety and efficacy of EMMM for odontogenic cysts and tumours.

\section{Authorship contribution}

TN: study design, data collection, manuscript preparation. NO: study design, data collection. DA, TO: data collection. SH: manuscript review.

\section{Conflicts of Interest}

None of the authors have any financial or other conflict of interest to declare. 


\section{References}

1. Boffano P, Ruga E, Gallesio C. Keratocystic odontogenic tumor (odontogenic keratocyst): Preliminary retrospective review of epidemiologic, clinical, and radiologic features of 261 lesions from University of Turin. J Oral Maxillofac Surg. 2010; 68: 2994-2999.

2. Seno S, Ogawal T, Shibayama M, et al. Endoscopic sinus surgery for the odontogenic maxillary cysts. Rhinology. 2009; 47: 305-309.

3. Manor E, Kachko L, Puterman MB, Szabo G, Bodner L. Cystic lesions of the jaws - a clinicopathological study of 322 cases and review of the literature. Int J Med Sci. 2012; 9: 20-26.

4. Hyo Y, Akisada T, Harada T. A Case of Keratocyst in the Maxillary Sinus. Jap Rhinol; 2007; 46: 97-101.

5. Di Pasquale P, Shermetaro C. Endoscopic removal of a dentigerous cyst producing unilateral maxillary sinus opacification on computed tomography. Ear Nose Throat J. 2006; 85: 747-748.

6. Ohki M. Transnasal Marsupialization Using Endoscopic Sinus Surgery for Treatment of Keratocystic Odontogenic Tumor in Maxillary Sinus. Case Rep Otolaryngol. 2012 2012: 1-4.

7. Kamel RH. Transnasal endoscopic medial maxillectomy in inverted papilloma.
Laryngoscope. 1995: 105: 847-853.

8. Wormald PJ, Ooi E, van Hasselt CA, Nair S. Endoscopic removal of sinonasal inverted papilloma including endoscopic medial maxillectomy. Laryngoscope. 2003; 113: 867-873.

9. Chen XB, Leong SC, Lee HP, Chong VF, Wang DY. Aerodynamic effects of rate inferior turbinate surgery on nasal flow - a computational fluid dynamics model. Rhinology. 2010; 48: 394-400

10. Heathcote KJ, Nair SB. The impact of modern techniques on the recurrence rate of inverted papilloma treated by endonasal surgery. Rhinology. 2009; 47: 339-344.

11. Modrzyński M. Hyaluronic acid gel in the treatment of empty nose syndrome. Am J Rhinol Allergy. 2011; 25: 103-106.

12. Nakayama T, Asaka D, Okushi T, Yoshikawa M, Moriyama H, Otori N. Endoscopic medial maxillectomy with preservation of inferior turbinate and nasolacrimal duct. Am J Rhinol Allergy. 2012; 26: 405-408.

13. Yasuoka T, Yonemoto K, Kato Y, Tatematsu N. Squamous cell carcinoma arising in a dentigerous cyst. J Oral Maxillofac Surg. 2000; 58: 900-905

14. Bodner L, Manor E, Shear M, van der Waal I. Primary intraosseous squamous cell carcinoma arising in an odontogenic cyst: a clinicopathologic analysis of 116 reported cases. J. Oral Pathol. Med. 2011 : 40: 733-738.

15. Gulbranson SH, Wolfrey JD, Raines JM McNally BP. Squamous cell carcinoma arising in a dentigerous cyst in a 16-month-old girl. Otolaryngol Head Neck Surg. 2002; 127 463-464.

16. Bodner L, Bar-Ziv J. Characteristics of bone formation following marsupialization of jaw cysts. Dentomaxillofac Radiol. 1998; 27: 166171.

Tsuguhisa Nakayama

Department of Otorhinolaryngology

Head and Neck Surgery

Dokkyo Medical University

880 Kita-Kobayashi, Mibu

Shimotsuga-gun

Tochigi 321-0293

Japan

Tel: $+81-282-87-2164$

Fax: +81-282-86-5928

E-mail: nakayama-t@jikei.ac.jp 